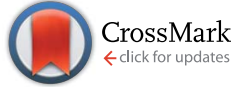

Cite this: Chem. Sci., 2016, 7, 3857

Received 28th January 2016 Accepted 27th February 2016

DOI: $10.1039 / \mathrm{c} 6 \mathrm{sc} 00428 \mathrm{~h}$

www.rsc.org/chemicalscience

\title{
Rational tuning of high-energy visible light absorption for panchromatic small molecules by a two-dimensional conjugation approach $\uparrow$
}

\author{
Bo He, $t^{a}$ Danylo Zherebetskyy, $\dot{t}^{\mathrm{b}}$ Hongxia Wang, $\dot{t}^{\mathrm{c}}$ Matthew A. Kolaczkowski, ${ }^{a}$ \\ Liana M. Klivansky, ${ }^{a}$ Tianwei Tan, ${ }^{c}$ Linwang Wang ${ }^{b}$ and Yi Liu*a
}

\begin{abstract}
We have demonstrated a rational two-dimensional (2D) conjugation approach towards achieving panchromatic absorption of small molecules. By extending the conjugation on two orthogonal axes of an electron acceptor, namely, bay-annulated indigo (BAl), the optical absorptions could be tuned independently in both high- and low-energy regions. The unconventional modulation of the highenergy absorption is rationalized by density functional theory (DFT) calculations. Such a 2D tuning strategy provides novel guidelines for the design of molecular materials with tailored optoelectronic properties.
\end{abstract}

\section{Introduction}

Organic semiconductors based on small molecules and conjugated polymers have attracted increasing interest for their applications in clean power generation, such as organic photovoltaics (OPVs) ${ }^{1}$ and dye-sensitized solar cells (DSSCs). ${ }^{2}$ For materials that best harness solar energies, their absorption profiles should have a large overlap with the visible region of the solar irradiance spectrum, which cover more than $60 \%$ of the solar photon flux density. ${ }^{3}$ It is therefore essential to design and synthesize single-component panchromatic materials that simultaneously absorb light over multiple visible wavelength ranges. A donor-acceptor approach is among the most rationalized strategies to tune optical properties of organic materials,${ }^{4}$ which can effectively reduce the optical bandgap towards the lower energy part of the spectrum by linking electron donors and acceptors in a linear conjugated backbone. Such an approach alone, however, is less effective in achieving panchromaticity, as the bathochromic shifts would leave void absorption spaces in the high-energy visible region. ${ }^{5}$ In donoracceptor conjugated polymers, this situation could be remedied by using two or more different types of co-monomers with sterically hindered side groups. In accordance with the

${ }^{a}$ The Molecular Foundry, Lawrence Berkeley National Laboratory, One Cyclotron Road, Berkeley, California, 94720, USA. E-mail: yliu@lbl.gov

${ }^{b}$ Materials Sciences Division, Lawrence Berkeley National Laboratory, One Cyclotron Road, Berkeley, California, 94720, USA

${ }^{c}$ Beijing Key Lab of Bioprocess, College of Life Science and Technology, Beijing University of Chemical Technology, Beijing 100029, China

$\dagger$ Electronic supplementary information (ESI) available: Experimental details, synthesis and characterization of 2D-BAIs, computational details. See DOI: 10.1039/c6sc00428h

\$ These authors contributed equally to this work. increased bulkiness, the co-planarity of the polymer main chain is decreased, resulting in hypochromic shifts that can fill in the high-energy void. ${ }^{6}$

Such a strategy is however not readily applicable for small molecules due to the absence of co-monomer units. As small molecule absorbers are advantageous over polymers in terms of ease of synthesis, less batch-to-batch variations and end-group contamination, ${ }^{7}$ it is imperative to devise a rational design rule that can merge discrete and localized absorptions to give panchromatic small molecules. Despite sporadic reports of small molecules with sub-optimal panchromatic properties, ${ }^{8}$ a rationalized approach is not available. ${ }^{5}$ Here we demonstrate a unique two-dimensional (2D) conjugation approach to afford panchromatic small molecules. The molecular system is based on a recently developed electron acceptor, the bay-annulated indigo (BAI) (Scheme 1a). ${ }^{9}$ By simply introducing thiophene units onto two orthogonal directions of a BAI core, independent tuning of optical absorptions in both low- and high-energy regions is realized.

Recently, old dyes have resurged as a potent class of building blocks for high performing optoelectronic materials. ${ }^{10}$ New families of $\pi$-conjugated systems obtained after chemical transformation of such dye cores have significantly broadened the materials scope of organic semiconductors. ${ }^{11}$ Among these dyes, indigo and related indigoids have received great attention due to their excellent ambipolar charge transport properties. ${ }^{\mathbf{1 2}}$ The direct use of indigo as a building block for organic semiconductors however remains infertile, due to interrupted conjugation across the central double bond and consequently, the $\pi$ electron resonance is localized within each oxindole unit. The chemical transformation by the double bay annulation step opens up a conjugation pathway that is orthogonal to the original long axis of indigo (Scheme 1a). The resulting BAIs 


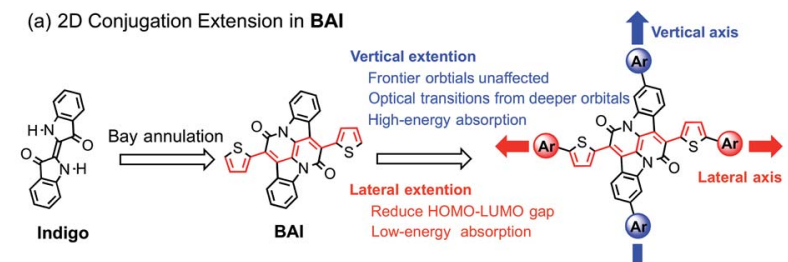

(b) List of BAls and Photos of the Respective Solutions (in $\mathrm{CHCl}_{3}$ )

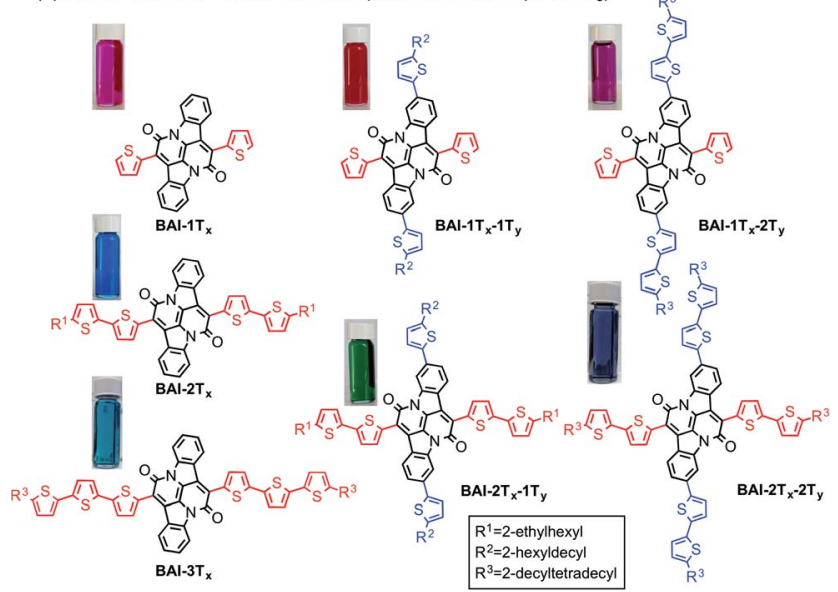

Scheme 1 (a) Illustration of 2D extension in BAI. (b) Structures of BAls and photos of the respective solutions.

behave as excellent electron acceptors, featuring low-lying LUMO energies and narrow optical bandgap. ${ }^{9,13}$ As observed in conventional donor-acceptor molecules, further extension of conjugation along the bay-annulation direction effectively lowers the optical bandgap towards the longer wavelength. On the other hand, aromatic units introduced on the orthogonal direction, that is, on the two phenylene rings of the parent indigo, are not fully delocalized and correspondingly induce new absorptions in shorter wavelength region. Theoretical calculations by density functional theory (DFT) have shed light on the nature of $2 \mathrm{D}$ electronic coupling and their impact on high-energy absorptions.

\section{Results and discussion}

Scheme 1b lists all the BAI derivatives with orthogonally functionalized aromatic substituents. For the ease of annotation, we arbitrarily defined the axis along the bay annulation direction as the "lateral" axis and the orthogonal axis defined by the two phenylene rings as the "vertical" axis. Thiophene-based substituent groups along the lateral or vertical axis of BAI were denoted as the suffix to BAI as $\boldsymbol{- n} \mathbf{T}_{\boldsymbol{x}}$ or $\boldsymbol{- n} \mathbf{T}_{\boldsymbol{y}}$, respectively, with $n$ indicating the number of thiophene units on each side of the BAI units. For example, BAI-2 $\mathbf{T}_{\boldsymbol{x}} \mathbf{- 2} \mathbf{T}_{\boldsymbol{y}}$ refers to the BAI derivative with two thiophene units at each end of the molecules on both lateral and vertical directions. Branched alkyl end groups with longer chain lengths were used in larger BAI derivatives to ensure good solubility in common organic solvents.

Lateral extension of $\mathbf{B A I - 1 T _ { \boldsymbol { x } }}$ was realized following the previously reported procedures. ${ }^{9}$ As shown in Scheme 2, Stille coupling between dibromide $\mathbf{1}^{9}$ and oligothiophene stannate gave BAI-2T $\mathbf{T}_{\boldsymbol{x}}$ and BAI-3T $\mathbf{T}_{\boldsymbol{x}}$ in good yields. For extension along the vertical axis, BAI bromide 3 was first obtained by reacting dibromoindigo $\mathbf{2}^{\mathbf{1 4}}$ with 2-thienylacetyl chloride in refluxing xylene, which was subjected to Stille coupling to give the corresponding BAI-1T $\mathbf{T}_{\boldsymbol{x}} \mathbf{- 1 T _ { \boldsymbol { y } }}$ and $\mathbf{B A I}-\mathbf{1} \mathbf{T}_{\boldsymbol{x}}-\mathbf{2} \mathbf{T}_{\boldsymbol{y}}$. Bromination of BAI-1T $_{\boldsymbol{x}}-\mathbf{1 T _ { \boldsymbol { y } }}$ and BAI-1T $\boldsymbol{T}_{\boldsymbol{x}}-\mathbf{2 T _ { \boldsymbol { y } }}$, followed by Stille coupling gave the 2D extended $\mathbf{B A I}-\mathbf{2} \mathbf{T}_{\boldsymbol{x}}-\mathbf{1} \mathbf{T}_{\boldsymbol{y}}$ and $\mathbf{B A I}-\mathbf{2} \mathbf{T}_{\boldsymbol{x}}-\mathbf{2} \mathbf{T}_{\boldsymbol{y}}$, respectively. The electrochemical properties of all BAI compounds were investigated (Fig. S3 in ESI $\dagger$ ) by cyclic voltammetry using a conventional three-electrode setup. The frontier orbital energies are estimated and summarized in Table 1 and Fig. S4. $\dagger$ All the BAI compounds have a LUMO energy level between -3.53 and $-3.69 \mathrm{eV}$, consistent with their electron accepting properties.

The optical absorption spectra of these BAI derivatives in $\mathrm{CHCl}_{3}$ solutions show distinct correlations in response to extended conjugation in two directions (Fig. 1, absorption normalized against the lowest energy peak, see Fig. S1† for the plots against extinction coefficients). The absorption spectrum of the purple BAI-1T $\mathbf{T}_{\boldsymbol{x}}$ solution shows double peaks at 542 and $579 \mathrm{~nm}$, characteristic for donor-acceptor molecules that correspond to the $\pi-\pi^{*}$ transition and intramolecular charge

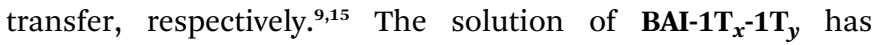
a reddish-purple color. A comparison of its absorption spectrum against that of $\mathbf{B A I - 1 T _ { x }}$ reveals a similar double peak feature, with the lowest energy peak red shifted by $10 \mathrm{~nm}$ (Fig. 1a). Notably, a new absorption peak emerges at $465 \mathrm{~nm}$ with a similar intensity. In the spectrum of the further vertically extended BAI-1T $\mathbf{T}_{\boldsymbol{x}}-\mathbf{2} \mathbf{T}_{\boldsymbol{y}}$ (Fig. 1a), the lowest energy peak remains at the same position as BAI-1T $\mathbf{T}_{\boldsymbol{x}} \mathbf{- 1 T _ { \boldsymbol { y } }}$. In the higher energy area, the peak at $465 \mathrm{~nm}$ observed in $\mathbf{B A I}-\mathbf{1 T}_{\boldsymbol{x}} \mathbf{- 1 T _ { \boldsymbol { y } }}$ now appears at 520 $\mathrm{nm}$. These spectroscopic changes indicate that vertical extension on BAI has a more dramatic effect on the absorption in the higher-energy part of the spectrum.

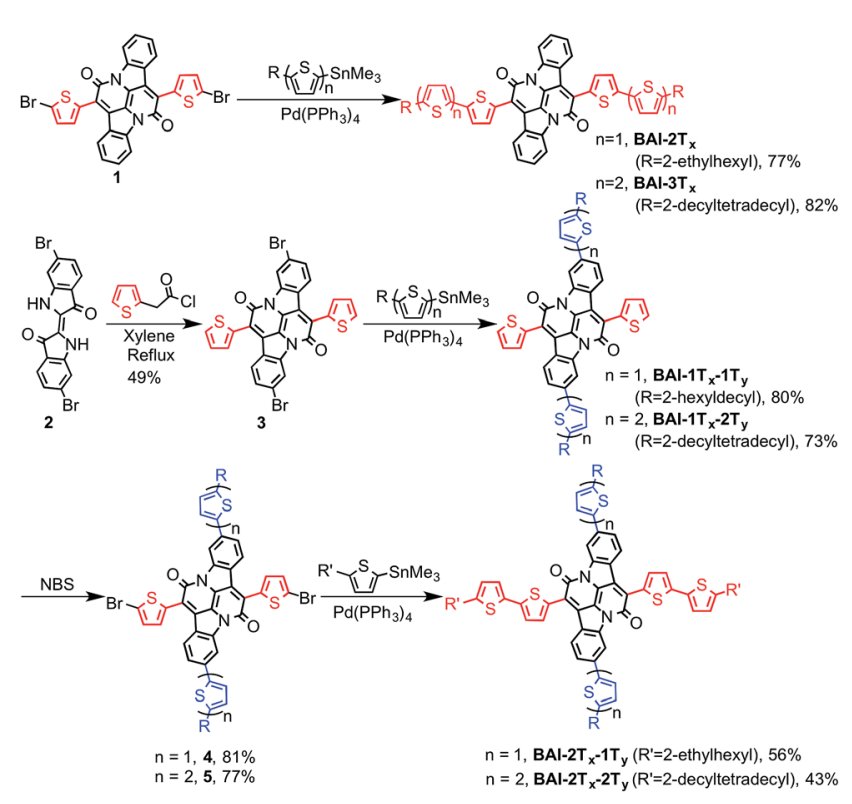

Scheme 2 Synthesis of BAI-based small molecules. 
Table 1 Summary of optical and electrochemical parameters of BAI derivatives

\begin{tabular}{|c|c|c|c|c|c|c|c|c|c|c|c|}
\hline Compd & \multicolumn{5}{|l|}{$\mathrm{UV}$ vis $^{a}$} & \multicolumn{6}{|c|}{ Cyclic voltammetry } \\
\hline BAI-2T $_{x}$ & 660 & 47600 & - & - & 745 & 1.66 & 0.39 & -1.13 & -5.19 & -3.67 & 1.52 \\
\hline BAI-3T $_{x}$ & 677 & 61700 & - & - & 800 & 1.55 & 0.23 & -1.11 & -5.03 & -3.69 & 1.34 \\
\hline BAI-1T $x_{x}-1 T_{y}$ & 589 & 30900 & 465 & 28800 & 630 & 1.97 & 0.51 & -1.24 & -5.31 & -3.56 & 1.75 \\
\hline BAI-2T $T_{x}-2 T_{y}$ & 677 & 44300 & 527 & 32000 & 759 & 1.63 & 0.36 & -1.12 & -5.16 & -3.68 & 1.48 \\
\hline
\end{tabular}

${ }^{a}$ In $\mathrm{CHCl}_{3} .{ }^{b}$ Wavelength of lowest energy absorption peak. ${ }^{c}$ Wavelength of new high energy absorption peak. ${ }^{d}$ Solution optical bandgap. ${ }^{e}$ Halfwave potentials $\left(v s . \mathrm{Fc} / \mathrm{Fc}^{+}\right) .{ }^{f}$ Solution electrochemical bandgap.
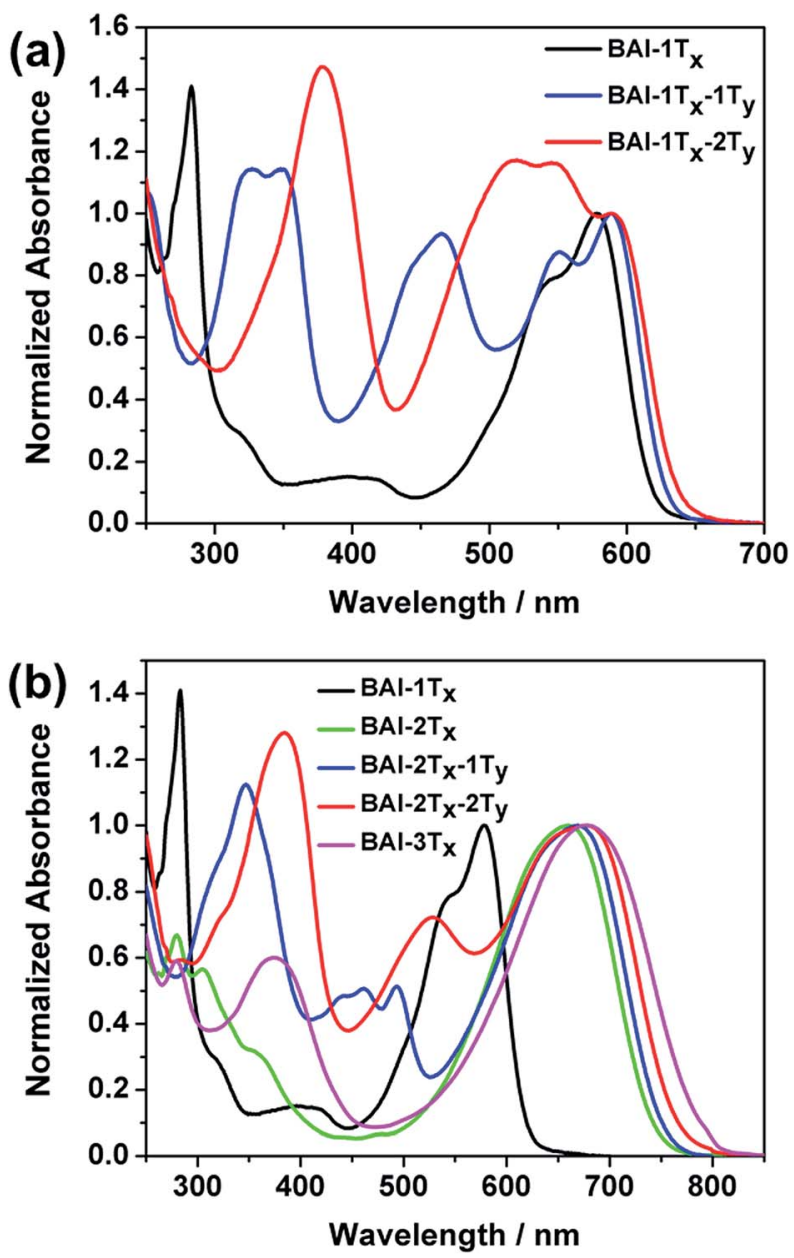

Fig. 1 Comparison of UV-Vis absorption spectra of (a) BAI-1T and the vertically expanded $\mathrm{BAI}-1 \mathrm{~T}_{x}-1 \mathrm{~T}_{y}$ and $\mathrm{BAI}-1 \mathrm{~T}_{x}-2 \mathrm{~T}_{y}$, (b) BAI-1T and the laterally expanded series BAI-2 $\mathrm{T}_{x}, \mathrm{BAI}-2 \mathrm{~T}_{x}-1 \mathrm{~T}_{y}$ and $\mathrm{BAI}-2 \mathrm{~T}_{x}-2 \mathrm{~T}_{y}$.

The impact of lateral conjugation extension on the optical properties is in sharp contrast to the vertical series. For the blue solution of BAI-2T $\mathbf{T}_{\boldsymbol{x}}$ and $\mathbf{B A I}-\mathbf{3} \mathbf{T}_{\boldsymbol{x}}$, the lowest energy absorptions peak at 660 and $697 \mathrm{~nm}$, respectively, corresponding to red shifts of 81 and $98 \mathrm{~nm}$ upon lateral extension of BAI-1T $\mathbf{T}_{\boldsymbol{x}}$ (Fig. 1b). In comparison to the vertically extended isomer BAI$\mathbf{1 T}_{\boldsymbol{x}} \mathbf{- 1} \mathbf{T}_{\boldsymbol{y}}$, the lateral extension leads to a more remarkable red shift while leaving a virtually void space between 400 and 500 $\mathrm{nm}$ in the spectrum. Introduction of one thiophene unit on the

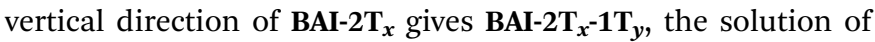
which displays a green colour and a similar lowest energy peak at $669 \mathrm{~nm}$. Encouragingly, new absorption peaks emerge to fill the valley area between 400 and $500 \mathrm{~nm}$. When the vertical conjugation is further extended by installing one more thio-

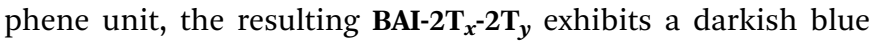
colour in solution. Consistent with the trend observed in Fig. 1a, the vertical extension imposes a small red shift of $8 \mathrm{~nm}$ for the lowest energy absorption, while the bluer region absorption becomes more prominent and undergoes a bathochromic shift to $527 \mathrm{~nm}$. Similar features are observed in the absorption spectra of thin films (Fig. S1 and S2 in ESI $\dagger$ ), except that peaks are broadened and the absorption onsets are shifted to longer wavelengths with respect to the corresponding solution spectra. The optical characteristics are summarized in Table 1.

Several trends from the optical studies are quite notable: (1) lateral extension of the $\pi$ conjugation results in significant red shift of lowest energy peaks; (2) vertical substitution with aromatic groups has minimum impact on the bandgap, instead complementary absorption peaks in the bluer region start to emerge; (3) the extinction coefficients of the lowest energy peaks increase as a result of lateral extension of conjugation, but are only slightly affected by vertical expansion. On the other hand, the new absorptions in the high-energy region induced by vertical thiophene substitutions have comparable extinction coefficients (of the order of $10^{4} \mathrm{M}^{-1} \mathrm{~cm}^{-1}$ ).

In order to understand the correlation between $2 \mathrm{D}$ functionalization of BAIs and their optoelectronic properties, DFT calculations were carried out to evaluate the frontier orbitals, related energies and the optical transitions. Generally, the lateral extension stabilizes the occupied orbitals and destabilizes the unoccupied orbitals, resulting in decreased energy gaps in between frontier orbitals (Fig. S5 $\dagger$ ). As shown in Fig. 2, the LUMOs of BAI- $\boldsymbol{n} \mathbf{T}_{\boldsymbol{x}}(n=1-3)$ are delocalized over the planar aromatic surfaces, including all the thiophenes and the two phenylene rings. The LUMOs are affixed to the lateral conjugation, and are barely affected by any extra thiophene units introduced vertically. On the other hand, confinement of conjugation in the lateral axis is universally observed for all the 


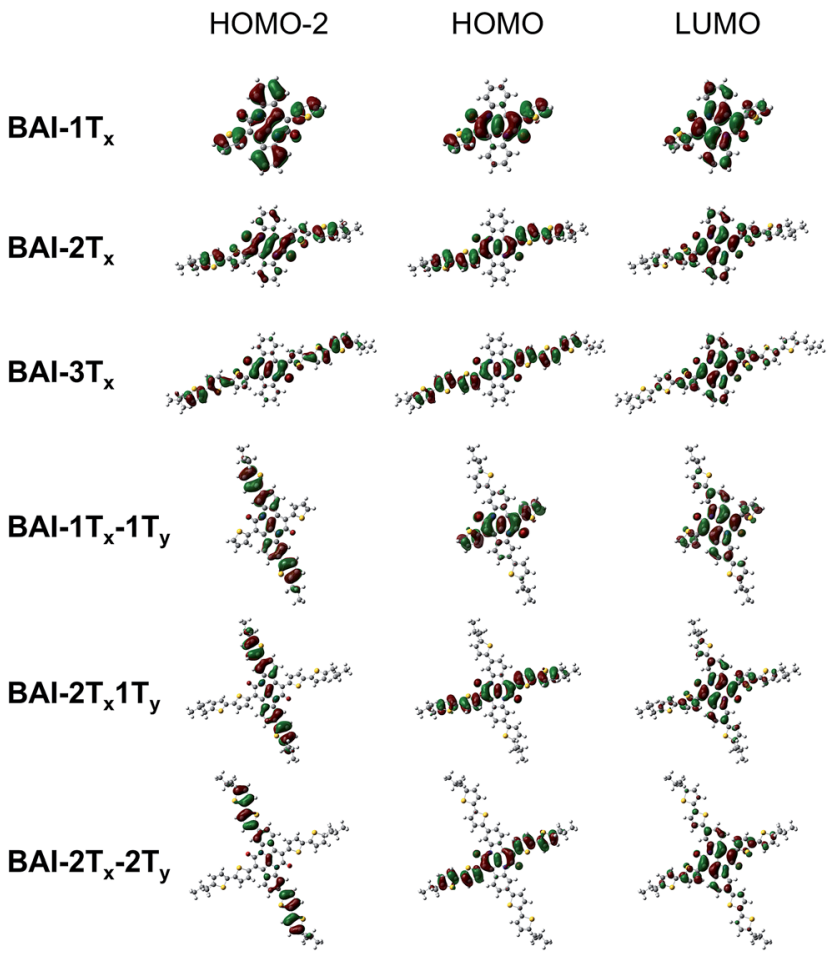

Fig. 2 Selected plots of frontier orbitals for the BAls. See Fig. S7† for a more complete set of plots.

HOMOs-neither the phenylene rings nor the vertically attached thiophene units have significant contributions to the HOMO orbital. Although vertical functionalization has minimal impact on HOMO and LUMO of these BAIs, it effectively stabilizes the deep orbitals (HOMO-1, HOMO-2, etc, and LUMO+1, LUMO+2, etc.), energetically squeezing them to be close to HOMO and LUMO (Fig. S5 $\dagger$ ), and rendering optical transitions that are essential for high-energy absorption. The calculated electronic absorption spectra using time-dependent DFT (TDDFT) show good agreement with the experimental spectra in terms of the relative positions of the absorption bands (Fig. S6†). Calculations on the optical transitions of BAIs reveal that while HOMO-1 to LUMO transitions are forbidden with close to zero oscillator strength, HOMO-1 to LUMO+1 transitions are allowed, leading to the intense high-energy peaks (in the 250-420 nm region). For the vertically substituted BAIs, optical transitions in the middle range (between the low- and high-energy peaks) are mainly from the HOMO-2 to LUMO transitions, together with smaller contributions from $\mathrm{HOMO}-4$ to LUMO and HOMO to LUMO+2 transitions. More detailed analysis of the compositions of transitions is shown in Table S1. $\dagger$ Fig. 2 compares selected plots of HOMO-2, HOMO and LUMO orbitals of BAIs. As indicated, the HOMO- 2 orbitals share great similarity to the HOMO orbitals for $\mathbf{B A I}-\boldsymbol{n} \mathbf{T}_{\boldsymbol{x}}(n=$ 1-3). When vertical thiophene substituents are introduced, the orbital distributions are drastically altered such that they are confined and delocalized vertically with very little contributions from the lateral aromatic constituents. Due to the alteration of the character of the deep molecular orbitals, it significantly increases (almost order of magnitude) the oscillator strength of the transitions in the center part of the spectrum (Table S1 $\dagger$ ). Overall, the HOMO-LUMO transition is minimally impacted by vertical extension, while transitions involving the deeper orbitals become the dominant feature in the shorter wavelength region.

\section{Conclusions}

In summary, we have demonstrated a $2 \mathrm{D}$ conjugation approach to achieve modular tuning of optical properties of BAI-based donor-acceptor molecules. As a result of aromatic substitutions along orthogonal molecular axis, absorptions in both the lowand high-energy regions of the visible spectrum can be finely tuned to achieve panchromatic absorption. Molecular modelling has revealed that the extensions along the lateral and vertical directions impart systematical changes on frontier orbitals and deep orbitals, respectively, which are responsible for the absorptions at different parts of the optical spectrum. The $2 \mathrm{D}$ conjugation motif has been applied in the synthesis of cruciform molecules ${ }^{16}$ for sensing ${ }^{17}$ and single-molecule conductance studies, ${ }^{18}$ and the modification of monomers ${ }^{19}$ for fine tuning electronic properties of the resulting polymers, but no insights have been provided towards tuning high-energy absorption profiles. We believe that the underlying principle should provide a more informed search for molecular materials with tailored optoelectronic properties.

\section{Acknowledgements}

This work was supported by Self-Assembly of Organic/Inorganic Nanocomposite Materials program, and was performed at the Molecular Foundry, both supported by the Office of Science, Office of Basic Energy Sciences, of the U.S. Department of Energy under Contract No. DE-AC02-05CH11231. H. W. and T. T. acknowledges the support from National Basic Research Program of China (973 program: 2013CB733600) and the National Nature Science Foundation of China (Grant No. 21436002, 21390202).

\section{Notes and references}

1 (a) Y.-J. Cheng, S.-H. Yang and C.-S. Hsu, Chem. Rev., 2009, 109, 5868-5923; (b) B. C. Thompson and J. M. J. Frechet, Angew. Chem., Int. Ed., 2008, 47, 58-77; (c) Y. Huang, E. J. Kramer, A. J. Heeger and G. C. Bazan, Chem. Rev., 2014, 114, 7006-7043; (d) C. Cabanetos, A. El Labban, J. A. Bartelt, J. D. Douglas, W. R. Mateker, J. M. J. Fréchet, M. D. McGehee and P. M. Beaujuge, J. Am. Chem. Soc., 2013, 135, 4656-4659; (e) K. A. Mazzio and C. K. Luscombe, Chem. Soc. Rev., 2015, 44, 78-90; (f) L. Dou, J. You, Z. Hong, Z. Xu, G. Li, R. A. Street and Y. Yang, Adv. Mater., 2013, 25, 6642-6671; (g) W. Chen, X. Yang, G. Long, X. Wan, Y. Chen and Q. Zhang, J. Mater. Chem. C, 2015, 3, 4698-4705; (h) B. Hu, M. Li, W. Chen, X. Wan, Y. Chen and Q. Zhang, RSC Adv., 2015, 5, 50137-50145.

2 (a) B. O'Regan and M. Gratzel, Nature, 1991, 353, 737-740; (b) M. Gratzel, Nature, 2001, 414, 338-344; (c) A. Hagfeldt, 
G. Boschloo, L. Sun, L. Kloo and H. Pettersson, Chem. Rev., 2010, 110, 6595-6663.

3 B. C. Popere, A. M. Della Pelle, A. Poe, G. Balaji and S. Thayumanavan, Chem. Sci., 2012, 3, 3093-3102.

4 (a) Z. G. Zhang and J. Wang, J. Mater. Chem., 2012, 22, 41784187; (b) K. Takimiya, I. Osaka and M. Nakano, Chem. Mater., 2014, 26, 587-593; (c) P. M. Beaujuge and J. M. J. Fréchet, J. Am. Chem. Soc., 2011, 133, 20009-20029.

5 J.-H. Yum, E. Baranoff, S. Wenger, M. K. Nazeeruddin and M. Gratzel, Energy Environ. Sci., 2011, 4, 842-857.

6 (a) P. M. Beaujuge, S. Ellinger and J. R. Reynolds, Nat. Mater., 2008, 7, 795-799; (b) P. M. Beaujuge and J. R. Reynolds, Chem. Rev., 2010, 110, 268-320; (c) W. T. Neo, C. M. Cho, Z. Shi, S.-J. Chua and J. Xu, J. Mater. Chem. C, 2016, 4, 28-32.

7 Z. B. Henson, K. Mullen and G. C. Bazan, Nat. Chem., 2012, 4, 699-704.

8 (a) S. Banala, T. Rühl, K. Wurst and B. Kräutler, Angew. Chem., Int. Ed., 2009, 48, 599-603; (b) J. M. Ball, N. K. S. Davis, J. D. Wilkinson, J. Kirkpatrick, J. Teuscher, R. Gunning, H. L. Anderson and H. J. Snaith, RSC Adv., 2012, 2, 6846-6853; (c) J. Qi, L. Ni, D. Yang, X. Zhou, W. Qiao, M. Li, D. Ma and Z. Y. Wang, J. Mater. Chem. C, 2014, 2, 2431-2438; (d) Z. Su, F. Hou, X. Wang, Y. Gao, F. Jin, G. Zhang, Y. Li, L. Zhang, B. Chu and W. Li, ACS Appl. Mater. Interfaces, 2015, 7, 2529-2534.

9 B. He, A. B. Pun, D. Zherebetskyy, Y. Liu, F. Liu, L. M. Klivansky, A. M. McGough, B. A. Zhang, K. Lo, T. P. Russell, L. Wang and Y. Liu, J. Am. Chem. Soc., 2014, 136, 15093-15101.

10 (a) M. J. Robb, S.-Y. Ku, F. G. Brunetti and C. J. Hawker, J. Polym. Sci., Part A: Polym. Chem., 2013, 51, 1263-1271; (b) T. Zhou, T. Jia, B. Kang, F. Li, M. Fahlman and Y. Wang, Adv. Energy Mater., 2011, 1, 431-439.

11 (a) K. Kotwica, P. Bujak, D. Wamil, M. Materna, L. Skorka, P. A. Gunka, R. Nowakowski, B. Golec, B. Luszczynska,
M. Zagorska and A. Pron, Chem. Commun., 2014, 50, 11543-11546; (b) M. K. Weclawski, M. Tasior, T. Hammann, P. J. Cywinski and D. T. Gryko, Chem. Commun., 2014, 50, 9105-9108; (c) K. Kotwica, P. Bujak, D. Wamil, A. Pieczonka, G. Wiosna-Salyga, P. A. Gunka, T. Jaroch, R. Nowakowski, B. Luszczynska, E. Witkowska, I. Glowacki, J. Ulanski, M. Zagorska and A. Pron, J. Phys. Chem. C, 2015, 119, 10700-10708; (d) J. Zhao, R. Li, W. Ai, D. Dong, J. Li, L. Chen, L. Xie, T. Yu and W. Huang, Chem. Asian J., 2016, DOI: 10.1002/asia.201501366, in press.

12 (a) E. D. Glowacki, G. Voss and N. S. Sariciftci, Adv. Mater., 2013, 25, 6783-6800; (b) M. Irimia-Vladu, E. D. Glowacki, P. A. Troshin, G. Schwabegger, L. Leonat, D. K. Susarova, O. Krystal, M. Ullah, Y. Kanbur, M. A. Bodea, V. F. Razumov, H. Sitter, S. Bauer and N. S. Sariciftci, Adv. Mater., 2012, 24, 375-380.

13 K. J. Fallon, N. Wijeyasinghe, N. Yaacobi-Gross, R. S. Ashraf, D. M. E. Freeman, R. G. Palgrave, M. Al-Hashimi, T. J. Marks, I. McCulloch, T. D. Anthopoulos and H. Bronstein, Macromolecules, 2015, 48, 5148-5154.

14 C. J. Cooksey, Molecules, 2001, 6, 736-769.

15 R. Stalder, J. G. Mei and J. R. Reynolds, Macromolecules, 2010, 43, 8348-8352.

16 (a) A. J. Zucchero, P. L. McGrier and U. H. F. Bunz, Acc. Chem. Res., 2010, 43, 397-408; (b) B. C. Tlach, A. L. Tomlinson, A. G. Ryno, D. D. Knoble, D. L. Drochner, K. J. Krager and M. Jeffries-El, J. Org. Chem., 2013, 78, 6570-6581.

17 M. A. Saeed, H. T. M. Le and O. Š. Miljanić, Acc. Chem. Res., 2014, 47, 2074-2083.

18 J. E. Klare, G. S. Tulevski, K. Sugo, A. de Picciotto, K. A. White and C. Nuckolls, J. Am. Chem. Soc., 2003, 125, 6030-6031.

19 (a) L. Ye, S. Zhang, L. Huo, M. Zhang and J. Hou, Acc. Chem. Res., 2014, 47, 1595-1603; (b) Y. Li, Acc. Chem. Res., 2012, 45, 723-733. 